\title{
Introduction
}

Francisco Javier Candel

\section{Introduction to XI Updating Course of Antimicrobials and Infectious Diseases}

Department of Clínical Microbiology and Infectious Diseases. IdISSC and IML Health Research Institutes. Hospital Clínico Universitario San Carlos. Madrid.

Last february, the XI Updating Course of Antimicrobials and Infectious Diseases was held at the Hospital Clínico San Carlos in Madrid. It is a scientific activity accredited by the Community of Madrid (Commission for Continuing Education of Health Professions at the Community of Madrid, file number 07-AFOC-00080.7/2021, 1 credit) and endorsed by the Spanish Society of Clinical Microbiology and Infectious Diseases (SEIMC), the Spanish Society of Chemotherapy (SEO) and the Madrid Society of Clinical Microbiology (SMMC). This year, the course was online edited and reached peaks of more than 1,500 conexions with continous mean over 750 . The audience consisted of multidisciplinar proffesionals of all specialties related to infection, the teachers made an update of the most relevant aspects on bacteriology, mycology and virology.

Current issue of the magazine includes summaries of the lectures given in the presential course. It also includes the questionnaire with the evaluations made by the students and a sheet of correct answers to being able to contrast the results. The supplement is divided into three headings. The first include a infectious diseases update with the most relevant information related to diagnostic techniques and management of infections presented during 2020, beyond COVID-19. It also includes an update on the Utility of level 2 laboratories in the National Antimicrobial Resistance Plan, an update of bioinformatics applied to the study of bacterial resistance and another on the treatment of multi-resistant microorganisms in hospital at home units. The second section provides a brief update on current antimicrobial pharmacotherapy and the third is a specific review of SARS-COV-2 infection, the value of diagnostic techniques, the integral management of infection, its complications and superinfections. 